\title{
Uso de sangue arterial e venoso no exame do equilíbrio ácido-básico de novilhos normais ou com acidose metabólica
}

\author{
Arterial and venous blood in the assessment of blood gas and acid-base status \\ in healthy steers or steers with metabolic acidosis
}

\author{
Maria Claudia Araripe Sucupira $^{1}$ Enrico Lippi Ortolani ${ }^{2}$
}

RESUMO

Doze novilhos foram utilizados para comparar os resultados hemogasométricos de sangue arterial e venoso. Esses animais foram divididos em três grupos idênticos, recebendo: 1- dieta balanceada, proporcionando ganho de peso de $600 \mathrm{~g} /$ d; 2- dieta de mantença em proteína e energia e 3-dieta com teores 20\% abaixo da mantença. Amostras de sangue arterial (artéria auricular caudal) e venoso (veia jugular) foram colhidas por 11 quinzenas consecutivas, num total de 132 amostras. Ao término do experimento, os animais dos grupos 2 e principalmente 3 apresentaram acidose metabólica moderada. Maiores valores de $\mathrm{pH}$ e de $\mathrm{pO}_{2}$ foram constatados no sangue arterial enquanto os teores de bicarbonato, $\mathrm{TCO}_{2}, \mathrm{BE}$ e $\mathrm{pCO}_{2}$ foram superiores no sangue venoso. Existiram altas correlações entre os valores do sangue arterial e venoso para o $\mathrm{pH}(r=$ $0,81)$, bicarbonato e $\mathrm{TCO}_{2}(r=0,94)$, sendo esta correlação média, mas significativa, no caso da $\mathrm{pCO}_{2}(r=0,56) e$ inexistente na $\mathrm{pO}_{2}(r=-0,06)$. Quanto maiores foram os valores de $\mathrm{pCO}_{2}$ maiores foram os teores de bicarbonato $(r=0,54) e$ $\mathrm{TCO}_{2}(r=0,56)$. Apesar das discrepancias de $\mathrm{pH}$, das concentrações de bicarbonato, $\mathrm{TCO}_{2}$, e mesmo de $\mathrm{pCO}_{2}$ obtidos no sangue venoso e arterial, é possivel estimar, adequadamente, a partir do primeiro esses valores nas amostras arteriais em novilhos normais e com moderado grau de acidose metabólica. Nos casos de alterações respiratórias, amostras de sangue arterial devem ser colhidas para determinação de $\mathrm{pO}_{2}$. A colheita de sangue venoso foi mais facilmente realizada que do sangue arterial.

Palavras-chave: hemogasometria, $\mathrm{pH}, \mathrm{pO}_{2}, \mathrm{pCO}_{2}, \mathrm{TCO}_{2}, \mathrm{BE}$, bicarbonato, bovino.

\begin{abstract}
Twelve steers were used to compare the blood gas and acid-base status. The animals were randomly divided in three equal groups according to the following diets: 1 -adequate, for gaining $600 \mathrm{~g} / \mathrm{head} / \mathrm{d} ; 2$ - protein and energy levels to meet the maintenence; 3 -protein and energy $20 \%$ below the maintenance levels. One hundred thirty-two arterial blood (caudal auricular artery) or venous blood (jugular vein) were continuously sampled for 11 fortnights. At the end of the experiment steers from groups 2 and principally 3 presented a moderate level of metabolic acidosis. Higher $\mathrm{pH}$ and $\mathrm{pO}_{2}$ levels were found in arterial blood; conversely, higher level of bicarbonate, $\mathrm{TCO}_{2}, \mathrm{BE}$ and $\mathrm{pO}_{2}$ levels were found in the venous blood. High correlation was found in the $p H(r=0.81)$, bicarbonate and $\mathrm{TCO}_{2}(r=0.94)$ between venous and arterial blood; median correlation but significant in the $\mathrm{pCO}_{2}(r=0.56)$ and lack of correlation in the $\mathrm{pO}_{2}(r=-$ 0.06). The higher the $\mathrm{pCO}_{2}$, the higher the bicarbonate $(r=0.54)$ and $\mathrm{TCO}_{2}(r=0.56)$ concentration. In spite of the small differences of $\mathrm{pH}$, bicarbonate, $\mathrm{TCO}_{2}$ and even $\mathrm{pCO}_{2}$ between venous and arterial blood the values of arterial blood can be predicted adequately from the venous blood either in healthy steers or steers with moderate metabolic acidosis. When respiratory changes are concerned arterial blood must be collected for evaluating $\mathrm{pO}_{2}$. It was easier to sample venous blood than arterial blood.
\end{abstract}

Key words: blood gas, $\mathrm{pH}, \mathrm{pO}_{2}, \mathrm{pCO}_{2}, \mathrm{TCO}_{2}, \mathrm{BE}$, bicarbonate, bovine.

${ }^{1}$ Médico Veterinário, PhD.

${ }^{2}$ Médico Veterinário, MSc, PhD, Professor do Departamento de Clínica Médica, Faculdade de Medicina Veterinária e Zootecnia, Universidade de São Paulo. Av. Prof. Dr. Orlando Marques n. 87, “Cidade Universitária”, 05508-000, São Paulo-SP, Brasil. E-mail: ortolani@usp.br. Autor para correspondência. 


\section{INTRODUÇÃO}

O exame hemogasométrico é de grande importância na avaliação do equilíbrio ácido-básico, o qual pode fornecer informações fundamentais para o diagnóstico e prognóstico de várias enfermidades dos bovinos. Dentre as disfunções do equilíbrio ácidobásico descritas, a mais comumente encontrada é a acidose metabólica, caracterizada por diminuição do $\mathrm{pH}$ e dos teores de bicarbonato sangüíneo. Encontrase tal desequilíbrio nos casos de acidose láctica ruminal, estados de cetoacidose, diarréia, insuficiência renal etc. (KANEKO et al., 1997; MARUTA \& ORTOLANI, 2002).

As principais determinações realizadas na hemogasometria são a do $\mathrm{pH}$, dos teores de dióxido de carbono no plasma $\left(\mathrm{TCO}_{2}\right)$, de bicarbonato e de excesso ou déficit de bases no sangue (BE), além das pressões parciais tanto de dióxido de carbono $\left(\mathrm{pCO}_{2}\right)$ quanto de oxigênio $\left(\mathrm{pO}_{2}\right)$. A hemogasometria pode ser realizada tanto em sangue venoso como em sangue arterial. Amostras de sangue arterial são preferidas em relação às venosas por causa da maior oxigenação do sangue e pelo fato de não serem modificadas em seus resultados hemogasométricos nos casos de estase do fluxo sangüíneo, em problemas respiratórios primários ou em pacientes mantidos sobre anestesia geral. Basicamente, as maiores diferenças entre os resultados hemogasométricos arteriais e venosos são os seguintes: maiores $\mathrm{pH}$ e $\mathrm{pO}_{2}$ nas amostras arteriais e maiores $\mathrm{pCO}_{2}$ e teores de bicarbonato e de $\mathrm{TCO}_{2}$ nas venosas; geralmente não se encontram diferenças nas concentrações de BE (DiBARTOLA, 1992; KANEKO et al. 1997).

Contudo, a despeito dessas diferenças nos resultados hemogasométricos o sangue venoso é rotineiramente utilizado por ser de mais fácil colheita que o arterial, e por oferecer resultados muito confiáveis nos casos de acidose metabólica (DiBARTOLA, 1992; KANEKO et al., 1997). Estudos comparativos foram realizados até o momento apenas nas espécies canina (van SLUIJS et al., 1983 e ILKIN et al., 1991), suína (HANNON et al., 1990), eqüina (SPEIRS 1980), necessitando ainda de comprovação, nas condições brasileiras, em bovinos.

O objetivo deste trabalho é comparar os resultados hemogasométricos obtidos em amostras de sangue arterial e venoso de novilhos hígidos ou com grau moderado de acidose metabólica.

\section{MATERIAL E MÉTODOS}

Foram utilizados 12 novilhos mestiços, hígidos, com cerca de 10 meses de idade e $170 \mathrm{~kg}$ de peso corporal no início do experimento. Os animais foram mantidos em baias individuais durante todo o experimento. Os bovinos foram divididos aleatoriamente em três grupos, de quatro animais, e submetidos aos seguintes tratamentos dietéticos, de acordo com os requerimentos propostos pelo NRC (1984) para gado de corte: 1- dieta controle, equilibrada em proteína e energia para o ganho de peso de $600 \mathrm{~g} / \mathrm{cab} / \mathrm{dia} ; 2$ - dieta com teores de proteína e energia suficientes apenas para a mantença; 3- dietas com $80 \%$ dos valores de mantença. Todos animais recebiam sal mineralizado comercial (Fosbov 20, Tortuga) e água ad libitum.

Foram colhidas amostras quinzenais de sangue arterial e venoso de todos os animais no decorrer de 11 quinzenas, num total de 132 pares de amostras, obtidas três horas após a alimentação matinal. O sangue arterial foi colhido da artéria auricular caudal, segundo descrição de FISHER et al. (1980), enquanto o sangue venoso foi obtido da veia jugular externa. Todos os cuidados para a manutenção da anaerobiose durante a colheita e conservação das amostras até a determinação hemogasométrica seguiram rigidamente as recomendações propostas por FISHER et al. (1980) e LISBOA et al. (2001). Cerca de uma hora após a colheita, as amostras foram processadas em analisador automático de $\mathrm{pH}$ e gases sangüíneos ${ }^{a}$. Das mesmas amostras sangüíneas, foram determinadas as concentrações de hemoglobina pelo método descrito por LISBOA et al. (2001).

Os dados foram comparados por teste de comparação de pares. O coeficiente de correlação e a regressão linear entre as variáveis também foram avaliados. Considerou-se uma correlação alta quando $\mathrm{r}>0,6$; média quando $0,3<\mathrm{r}<0,6$; e baixa quando $\mathrm{r}<0,3$ (LITTLE \& HILLS, 1978).

\section{RESULTADOS}

Ao término do experimento, os novilhos do $1^{\circ}$ grupo ganharam individualmente ao redor $100 \mathrm{~kg}$ de peso vivo, os do $2^{\circ}$ mantiveram seu peso e os animais do $3^{\circ}$ grupo perderam $18,5 \mathrm{~kg}$.

Os animais do grupo controle apresentaram no decorrer de todo o experimento condições normais de equilíbrio ácido-básico. Já a redução do oferecimento de energia e de proteína dietética provocou, principalmente, no grupo que recebia dieta mais carente um quadro de acidose metabólica moderada, com queda no pH (menor valor foi de 7,16 ) e na concentração sangüínea de bicarbonato (menor teor foi de 13 $\mathrm{mM})$. 
Embora o pH sangüíneo médio fosse menor $(7,34 \pm 0,06 \times 7,38 \pm 0,06 ; p<0,0001)$ no sangue venoso que no arterial existiu uma alta correlação positiva $(\mathrm{r}=0,88)$ entre estas variáveis (Figura 1). O sangue arterial apresentou menores teores de bicarbonato sangüíneo $(22,9 \pm 3,8 \mathrm{mM} \mathrm{X}$ $23,7 \pm 3,9 \mathrm{mM} ; \mathrm{p}<0,05)$ e do total de dióxido de carbono no plasma ( $\left.\mathrm{TCO}_{2}\right)(24 \pm 3,8 \mathrm{mM} \mathrm{X} 25 \pm$ 3,9 mM; $\mathrm{p}<0,046)$. Tanto para os teores de bicarbonato como de $\mathrm{TCO}_{2}$ ocorreram altas correlações positivas entre o sangue venoso e arterial $(\mathrm{r}=0,94 \mathrm{em}$ ambos os casos; figuras 2 e 3 , respectivamente). Não existiu diferença entre as médias de excesso ou déficit de bases no sangue (BE) $(-1,2 \pm 4,2 \mathrm{mM} \mathrm{X}-1,3 \pm 4,3 \mathrm{mM} ; \mathrm{p}>0,93)$. A pressão de dióxido de carbono $\left(\mathrm{pCO}_{2}\right)$ foi maior no sangue venoso que no arterial $(45,2 \pm 4,4 \mathrm{mmHg} X$ $39,8 \pm 4,2 \mathrm{mmHg} ; \mathrm{p}<0,0001)$. Existiu correlação positiva entre a $\mathrm{pCO}_{2}$ venosa e arterial $(\mathrm{r}=0,56$ e $\mathrm{p}<$ 0,0001; Figura 4). A pressão de oxigênio (pO ) foi extremamente superior no sangue arterial $\left(91,7^{2} \pm 12\right.$ $\mathrm{mmHg} X 35,8 \pm 6 \mathrm{mmHg} ; \mathrm{p}<0,0001)$, não existindo correlação desta variável entre os dois diferentes tipos de sangue $(r=-0,06)$.

Quanto maiores as $\mathrm{pCO}_{2}$, detectadas tanto no sangue venoso como no arterial, maiores foram as concentrações de bicarbonato $(r=0,54 \mathrm{p}<0,0001$, Figura 5) e de $\mathrm{TCO}_{2}(\mathrm{r}=0,56 ; \mathrm{p}<0,0001$, Figura 6) no sangue. Não existiram diferenças nas concentrações venosas e arteriais de hemoglobina $(\mathrm{p}>0,82)$.

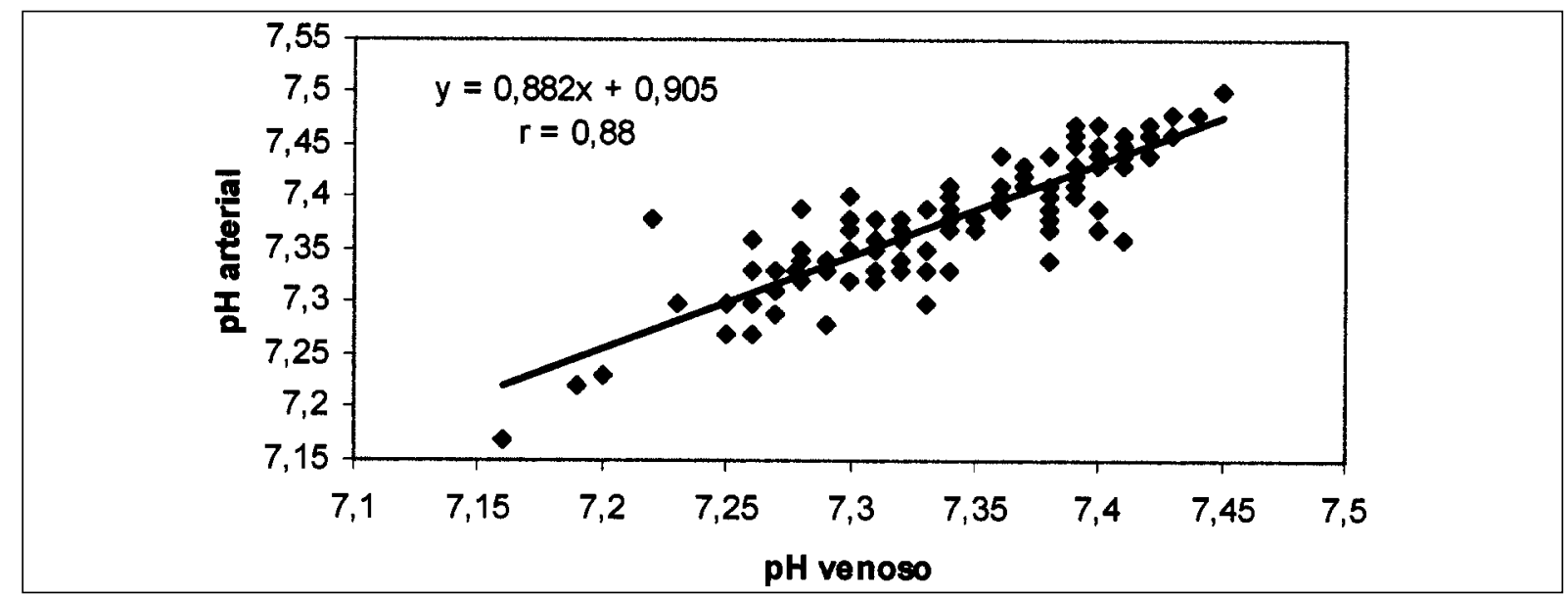

Figura 1 - Relação entre o $\mathrm{pH}$ do sangue venoso e o $\mathrm{pH}$ do sangue arterial de novilhos mestiços recebendo dieta equilibrada em proteína e energia para ganho de $600 \mathrm{~g} / \mathrm{an} / \mathrm{dia}$, dieta com teores de proteína e energia suficientes para mantença ou dieta com $80 \%$ dos valores de mantença durante 11 quinzenas.

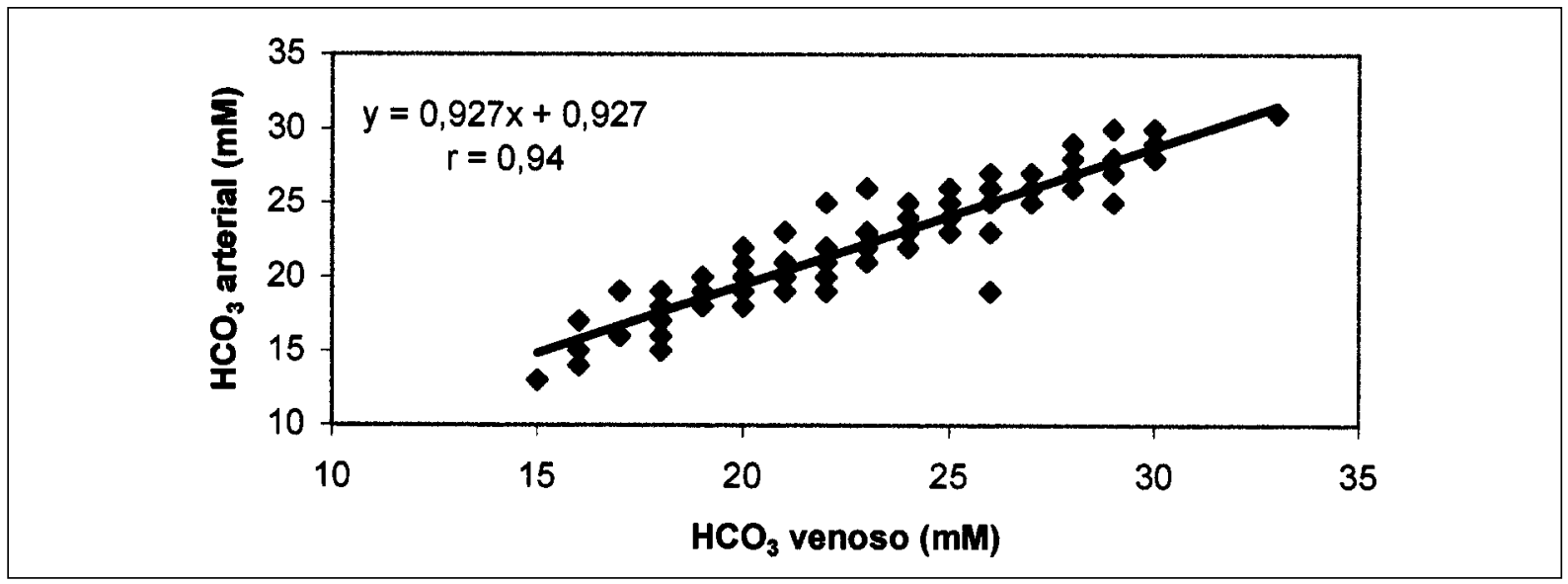

Figura 2 - Relação entre as concentrações de $\mathrm{HCO}_{3}(\mathrm{mM})$ no sangue venoso e no sangue arterial de novilhos mestiços recebendo dieta equilibrada em proteína e energia para ganho de $600 \mathrm{~g} / \mathrm{an} /$ dia, dieta com teores de proteína e energia suficientes para mantença ou dieta com $80 \%$ da mantença durante 11 quinzenas.

Ciência Rural, v. 33, n. 5, set-out, 2003. 


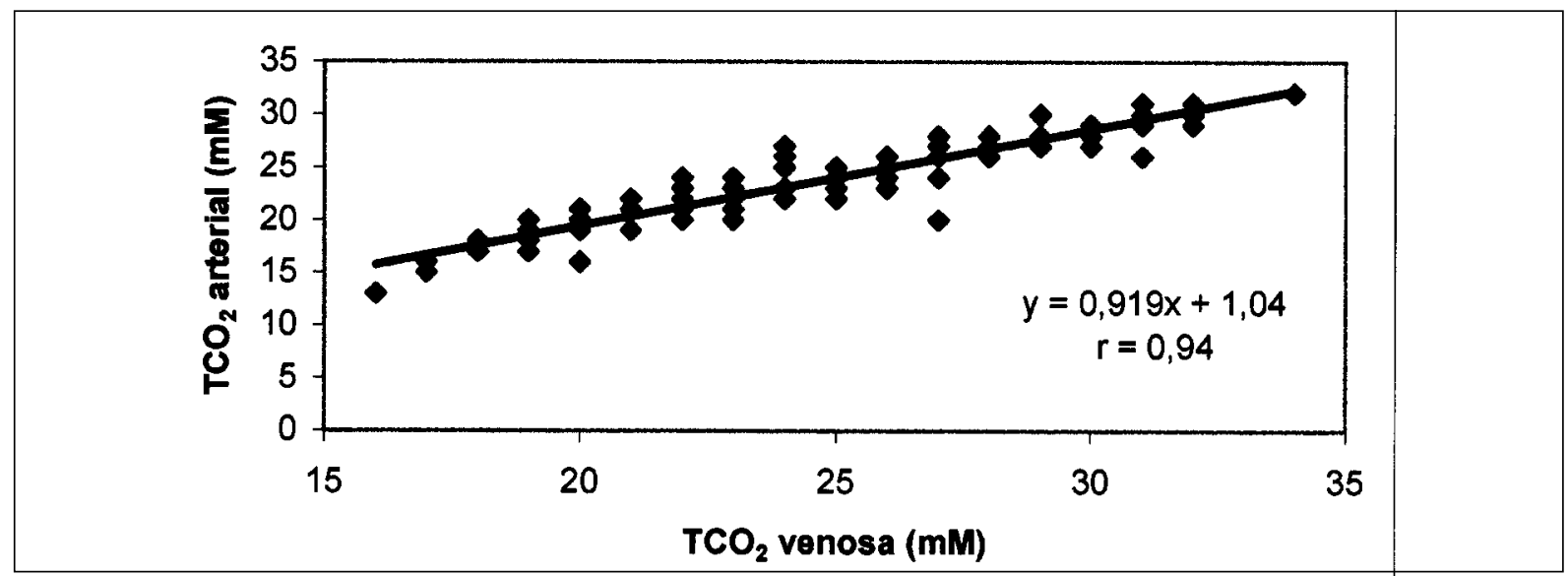

Figura 3 - Relação entre as concentrações de $\mathrm{TCO}_{2}(\mathrm{mM})$ no sangue venoso e no sangue arterial de novilhos mestiços recebendo dieta equilibrada em proteína e energia para ganho de $600 \mathrm{~g} / \mathrm{an} /$ dia, dieta com teores de proteína e energia suficientes para mantença ou dieta com $80 \%$ da mantença durante 11 quinzenas.

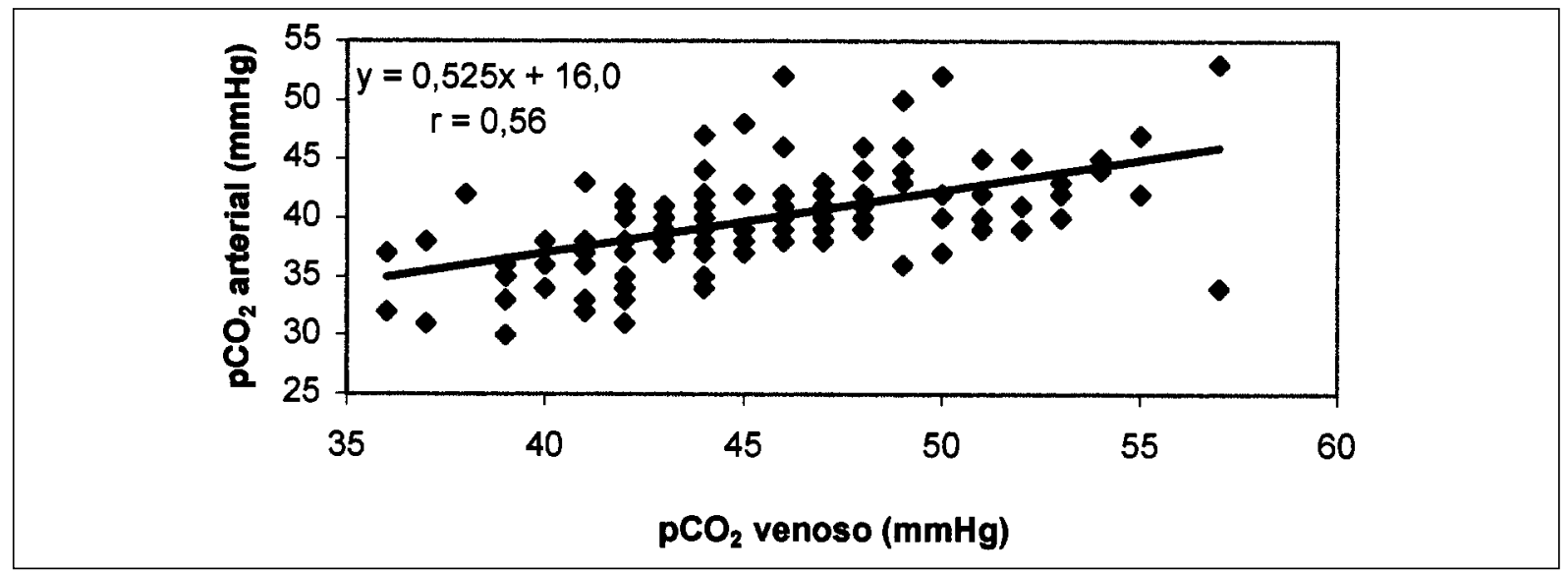

Figura 4 - Relação entre a $\mathrm{pCO}_{2}(\mathrm{mmHg})$ no sangue venoso e no sangue arterial de novilhos mestiços recebendo dieta equilibrada em proteína e energia para ganho de $600 \mathrm{~g} / \mathrm{an} / \mathrm{dia}$, dieta com teores de proteína e energia suficientes para mantença ou dieta com $80 \%$ da mantença durante 11 quinzenas.

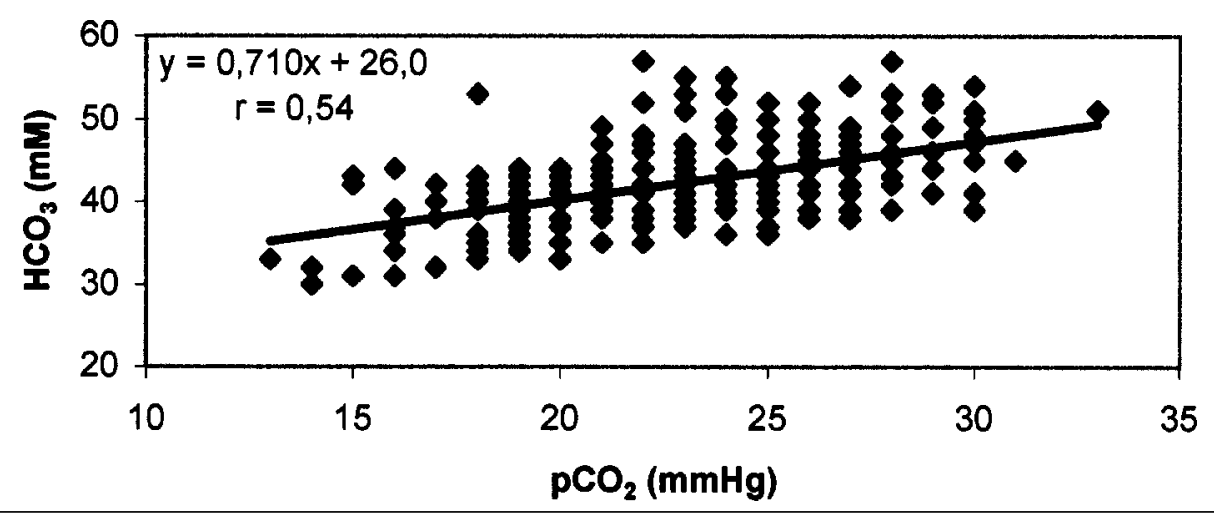

Figura 5 - Relação entre $\mathrm{HCO}_{3}(\mathrm{mM})$ e $\mathrm{pCO}_{2}(\mathrm{mmHg})$ de novilhos mestiços recebendo dieta equilibrada em proteína e energia para ganho de $600 \mathrm{~g} / \mathrm{an} / \mathrm{dia}$, dieta com teores de proteína e energia suficientes para mantença ou dieta com $80 \%$ da mantença durante 11 quinzenas.

Ciência Rural, v. 33, n. 5, set-out, 2003. 


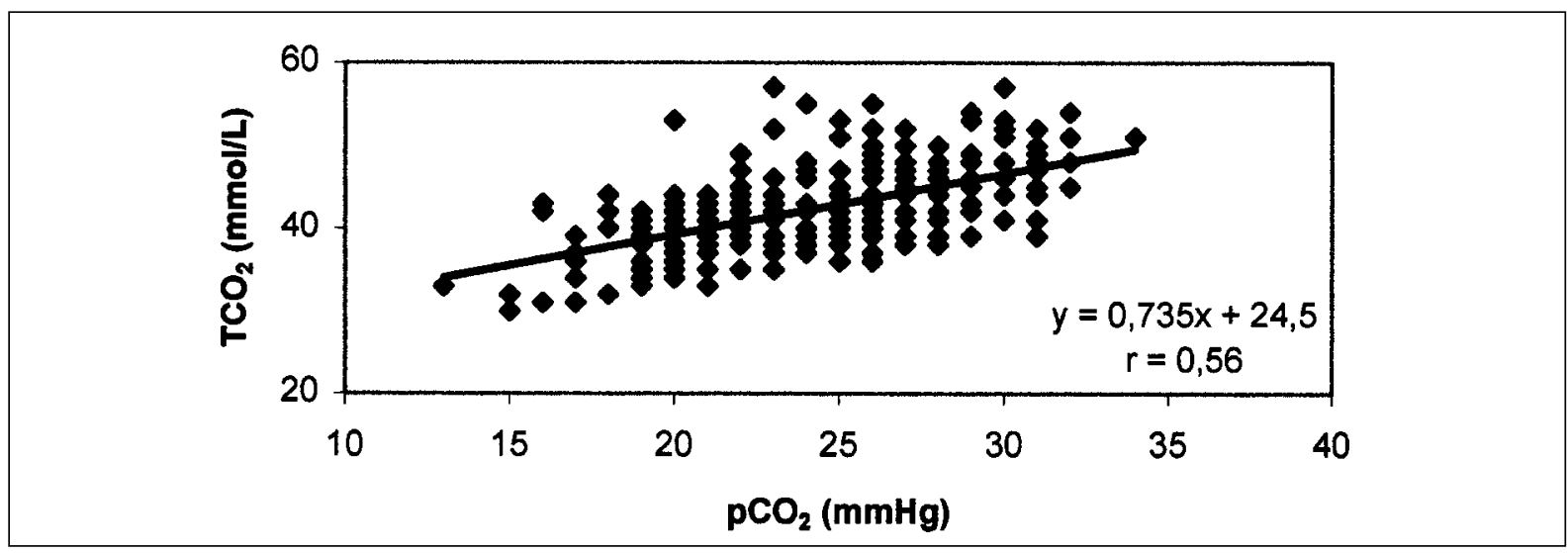

Figura 6 - Relação entre $\mathrm{TCO}_{2}(\mathrm{mmol} / \mathrm{L})$ e $\mathrm{pCO}_{2}(\mathrm{mmHg})$ de novilhos mestiços recebendo ou dieta equilibrada em proteína e energia para ganho de $600 \mathrm{~g} / \mathrm{cab} / \mathrm{dia}$, ou dieta com teores de proteína e energia suficientes para mantença ou ainda dieta com déficit de $20 \%$ com relação à mantença durante 11 quinzenas.

\section{DISCUSSÃO}

Idêntico ao que foi descrito em cães (van SLUIJS et al., 1983 e ILKIN et al., 1991), suínos (HANNON et al., 1990) e eqüinos (SPEIRS, 1980), o pH sangüíneo detectado foi inferior no sangue venoso, ocorrendo o inverso em relação aos teores de bicarbonato, de $\mathrm{TCO}_{2}$ e das pressões parciais de $\mathrm{CO}_{2}$. Segundo KANEKO et al. (1997), este maior teor de bicarbonato no sangue venoso é decorrente do próprio aumento na geração de $\mathrm{CO}_{2}$ da respiração celular, já que parte deste gás pode ser transportado na forma de bicarbonato. De fato, comprovou-se aqui que quanto maiores foram as pressões de $\mathrm{CO}_{2}$ maiores os teores de bicarbonato e de $\mathrm{TCO}_{2}$ no sangue (Figuras 5 e 6 ).

Semelhante ao que é classicamente descrito, a diferença entre as concentrações de $\mathrm{TCO}_{2}$ e bicarbonato, neste trabalho, ficou entre 1 e $2 \mathrm{mM}$ a favor do primeiro, indicando que tanto na colheita como na conservação as amostras foram mantidas em condições adequadas de anaerobiose (DiBARTOLA, 1992; KANEKO et al., 1997).

As concentrações de excesso de base (BE) não foram diferentes $(p>0,93)$ entre o sangue venoso $e$ arterial. Isto também foi relatado por DiBARTOLA (1992) e KANEKO et al. (1997), porém sem justificar esta semelhança. Especula-se que, pelo fato do BE ser calculado a partir do $\mathrm{pH}$ sangüíneo e da $\mathrm{pCO}_{2}$ e também considerando-se que seus valores se comportam de forma oposta no sangue arterial e venoso, provavelmente exista uma equiparação que torna o $\mathrm{BE}$ semelhante nas mais diversas condições de equilíbrio ácido-básico.

Como era de se esperar, os maiores valores de $\mathrm{pCO}_{2}$ foram constatados no sangue venoso. Embora a correlação não fosse alta entre a $\mathrm{pCO}_{2}$ venosa e arterial sua significância foi bastante expressiva $(r=0,56 ; p<0,0001$, Figura 4). Este resultado indica que a estimativa do $\mathrm{pCO}_{2}$ arterial a partir do valor venoso é possível e confiável em bovinos hígidos e com moderada acidose metabólica.

Os presentes resultados confirmam que, na estimativa do $\mathrm{pO}_{2}$, o sangue venoso não deve ser utilizado, já que os valores foram muito discrepantes e não houve correlação significativa entre as amostras venosas e arteriais $(r=-0,06)$.

As pequenas discrepâncias existentes nos resultados de $\mathrm{pH}$ (Figura 1), nas concentrações de bicarbonato (Figura 2) e $\mathrm{TCO}_{2}$ (Figura 3) sangüíneos entre o sangue venoso e arterial podem ser facilmente corrigidos através das equações de regressão apresentadas, já que existiram altas correlações entre os resultados obtidos. O conjunto desses resultados permite afirmar que o sangue venoso pode ser rotineiramente utilizado para substituir o arterial nos exames hemogasométricos, exceto quando se deseja analisar alterações na $\mathrm{pO}_{2}$ decorrentes de alterações respiratórias.

A colheita de sangue na artéria auricular caudal não foi de difícil execução, mesmo assim foi um processo mais trabalhoso e lento que a obtenção de amostras na veia jugular, as quais eram colhidas mais rapidamente e em maior volume.

Conclui-se que os valores de $\mathrm{pH}$, bicarbonato, $\mathrm{TCO}_{2}$ e BE correlacionam altamente com o sangue arterial de bovinos, existindo uma correlação média para o $\mathrm{pCO}_{2}$ e ausência de correlação para o $\mathrm{pO}_{2}$. Estes resultados indicam que o sangue venoso pode refletir precisamente o estado ácido-básico de bovinos hígidos ou com moderada acidose metabólica.

Ciência Rural, v. 33, n. 5, set-out, 2003. 


\section{AGRADECIMENTOS}

Agradecimentos às técnicas Regina S. Mirandola e Clara S. Mori pelo apoio nas determinações laboratoriais; ao funcionário Aguinaildo Arcanjo dos Santos pela manutenção dos animais, aos pós-graduandos Alexandre C. Antonelli, Celso A. Maruta, Pierre C. Soares, Sandra S. Kitamura pelo auxílio nas várias etapas do experimento.

\section{FONTES DE AQUISIÇÃO}

a - Hemogasômetro ABL 05, Radiometer, Copenhagen.

\section{REFERÊNCIAIS BIBLIOGRÁFICAS}

DiBARTOLA, S.P. Fluid therapy in small animal practice. Philadelphia : Saunders, 1992. 720p.

FISHER, E.W.; SIBARTIE, D.; GRIMSHAW, W.T.R. A comparison of the $\mathrm{pH}, \mathrm{pCO}_{2}, \mathrm{pO}_{2}$ and total $\mathrm{CO}_{2}$ content in blood from the brachial and caudal auricular arteries in normal cattle. British Veterinary Journal, v.136, p.496-499, 1980.

HANNON, J.P.; BOSSONE, C.A.; WADE, C.E. Normal physiological values for conscious pigs used in biomedical research. Laboratory Animal Science, v.40, p.293-299, 1990.

ILKIN, J.E.; ROSE, R.J.; MARTIN, C.A. A comparison of simultaneously collected arterial, mixed venous, jugular venous and cephalic venous blood samples in the assessment of blood- gas and acid-base status in the dog. Journal of Veterinary Internal Medicine, v.5, p.294-298, 1991.

KANEKO, J.J.; HARVEY, J.W.; BRUSS, M.L. Clinical biochemistry of domestic animals. 5.ed. San Diego : Academic, 1997. 932p.

LISBOA, J.A.N. et al. Tempo de viabilidade de amostras de sangue venoso bovino destinadas ao exame hemogasométrico quando mantidas sob conservação em água gelada. Ciência Rural, v.31, n.2, p.271-276, 2001.

LITTLE, T.M.; HILLS, F.J. Agricultural experimentation design and analysis. New York : John Wiley \& Sons, 1978. 305p.

MARUTA, C.A.; ORTOLANI, E.L. Susceptibilidade de bovinos das raças Jersey e Gir à acidose láctica ruminal: II - Acidose metabólica e metabolização do lactato-L. Ciência Rural, v.32, n.1, p.61-65, 2002.

NATIONAL RESEARCH COUNCIL. Committe on Animal Nutrition. Nutrient requeriments of beef cattle. 6.ed. Washington : National Academy of Science, 1984. 90p.

SPEIRS, V.C. Arteriovenous and arteriocentral venous relationships for $\mathrm{pH}, \mathrm{pCO}_{2}$ and actual bicarbonate in equine blood samples. American Journal Veterinary Research, v.41, p.199203, 1980.

VAN SLUIJS, F.J. et al. Capillary and venous blood compared with arterial blood in the measurement of acid-base and blood gas status of dogs. American Journal Veterinary Research, v.44, p.459-462, 1983. 